\title{
Linear Prediction Approach for Accurate Dual-Channel Sine-Wave Parameter Estimation in White Gaussian Noise
}

$\overline{\text { Hing-Cheung So and Zhenhua Zhou }}$

The problem of sinusoidal parameter estimation at two channels with common frequency in white Gaussian noise is addressed. By making use of the linear prediction property, an iterative linear least squares (LLS) algorithm for accurate frequency estimation is devised. The remaining parameters are then determined according to the LLS fit with the use of the frequency estimate. It is proven that the variance of the frequency estimate achieves Cramér-Rao lower bound at sufficiently small noise conditions.

Keywords: Sinusoidal parameter estimation, linear prediction, least squares, dual-channel.

\section{Introduction}

In this letter, we address the problem of finding the sinusoidal parameters from noisy observations received at two channel outputs. This has applications such as velocity and size estimation in phase Doppler anemometry and impedance measurement [1]. In each channel, the noise-free signal is a real sinusoid with a DC offset. As the frequency is common, there are seven unknowns of interest, namely, one frequency as well as two amplitudes, phases, and DC offsets. In [2], [3], the nonlinear least squares (NLS) estimator is proposed, which first finds the frequency through a one-dimensional (1D) search, followed by a linear least squares (LLS) fit of the remaining parameters. Although its mean square error (MSE) performance can attain Cramér-Rao lower bound (CRLB) [2] in the presence of white Gaussian noise, there is no guarantee

Manuscript received Nov. 20, 2011; revised Jan. 10, 2012, accepted Feb. 7, 2012.

Hing-Cheung So (phone: +86 8523442 7780, hcso@ee.cityu.edu.hk) and Zhenhua Zhou (corresponding author, zhenhzhou2@student.cityu.edu.hk) are with the Department of Electronic Engineering, City University of Hong Kong, Hong Kong, China.

http://dx.doi.org/10.4218/etrij.12.0211.0488 of obtaining the global solution. It is because the 1D search in the multi-modal surface is typically performed in two steps. First, we evaluate the NLS objective function at a number of grid points, and the coarse frequency estimate is given by the grid point corresponding to the lowest function value. A local search based on a numerical method with the coarse estimate being the initial guess is then employed in the second step. When the number of grid points is not sufficiently large, it is possible that the coarse estimate corresponds to a local minimum, which results in a large frequency estimation error.

In this work, the linear prediction (LP) approach is utilized to develop an iterative LLS algorithm for frequency estimation using dual-channel data so that no $1 \mathrm{D}$ search is required. The remaining parameters are then solved straightforwardly according to an LLS procedure. It is worth pointing out that we have recently utilized this methodology in parameter estimation for wave equation [4]. Nevertheless, the LP relation in this work is different from that of [4], and we have also extended the idea to multiple channels. Moreover, it is proven that the variance of the frequency estimate attains the CRLB at sufficiently high signal-to-noise ratio (SNR) conditions.

\section{Proposed Method}

The dual-channel sinusoidal signal model is

$$
x_{i}(n)=s_{i}(n)+v_{i}(n), \quad i=1,2,
$$

where

$$
s_{i}(n)=\alpha_{i} \cos \left(\omega n+\phi_{i}\right)+C_{i}, \quad n=1, \cdots, N .
$$

Denoting the amplitude, initial phase, and DC offset at the $i$-th 
channel are $\alpha_{i}>0, \phi_{i} \in[0,2 \pi)$, and $C_{i}$, respectively, while $\omega \in[0, \pi)$ is the common frequency, and they are the unknown parameters to be estimated. For simplicity but without loss of generality, we assume that the additive noises $v_{1}(n)$ and $v_{2}(n)$ are uncorrelated white Gaussian processes with variances $\sigma_{1}^{2}$ and $\sigma_{2}^{2}$, respectively. Here, we assume their ratio, denoted by $r=\sigma_{1}^{2} / \sigma_{2}^{2}$, is known a priori.

According to [5], $s_{i}(n)$ satisfies the following LP property:

$$
s_{i}(n)-s_{i}(n-3)-\rho\left(s_{i}(n-1)-s_{i}(n-2)\right)=0
$$

for $n=4,5, \cdots, N$, where $\rho=(2 \cos (\omega)+1)$ is called the LP coefficient, which is linear in (3). Using (3), an LP error vector for the $i$-th channel, denoted by $\mathbf{e}_{i}$, is established as

$$
\mathbf{e}_{i}=\mathbf{x}_{4 i}-\mathbf{x}_{1 i}-\tilde{\rho}\left(\mathbf{x}_{3 i}-\mathbf{x}_{2 i}\right), \quad i=1,2,
$$

where $\mathbf{x}_{m i}=\left[x_{i}(m) x_{i}(m+1) \cdots x_{i}(m+N-4)\right]^{T}, m=1,2,3,4$, and $\tilde{\rho}$ is the variable for $\rho$. Stacking the error vectors as $\mathbf{e}=\left[\mathbf{e}_{1}^{T} \mathbf{e}_{2}^{T}\right]^{T}$, the LLS estimate of $\rho$, denoted by $\hat{\rho}$, is

$$
\hat{\rho}=\arg \min _{\tilde{\rho}} \mathbf{e}^{T} \mathbf{W e}=\frac{\mathbf{z}_{2}^{T} \mathbf{W} \mathbf{z}_{1}}{\mathbf{z}_{2}^{T} \mathbf{W} \mathbf{z}_{2}},
$$

where $\mathbf{z}_{1}=\left[\begin{array}{lll}\mathbf{x}_{41}^{T}-\mathbf{x}_{11}^{T} & \mathbf{x}_{42}^{T}-\mathbf{x}_{12}^{T}\end{array}\right]^{T}, \mathbf{z}_{2}=\left[\begin{array}{ll}\mathbf{x}_{31}^{T}-\mathbf{x}_{21}^{T} & \mathbf{x}_{32}^{T}-\mathbf{x}_{22}^{T}\end{array}\right]^{T}$, and $\mathbf{W}$ is the weighting matrix and its optimal form is determined as [6]

$$
\mathbf{W}=\left[\left.E\left\{\mathbf{e e}^{T}\right\}\right|_{\tilde{\rho}=\rho}\right]^{-1}=\operatorname{blkdiag}\left(\mathbf{W}_{S 1}, \mathbf{W}_{S 2}\right),
$$

where

$$
\begin{aligned}
& \mathbf{W}_{S i}^{-1}=\left.E\left\{\mathbf{e}_{i} \mathbf{e}_{i}^{T}\right\}\right|_{\tilde{\rho}=\rho} \\
& =\operatorname{Toeplitz}\left(\left[\begin{array}{llllllll}
2\left(\rho^{2}+1\right) & -\rho^{2}-2 \rho & 2 \rho & -1 & 0 & \cdots & 0
\end{array}\right]\right) \sigma_{i}^{2}
\end{aligned}
$$

for $i=1,2$. The $E$ represents the expectation operator, blkdiag( $(\cdot)$ denotes the block diagonal matrix, and Toeplitz $\left(\mathbf{u}^{T}\right)$ stands for the Toeplitz matrix with $\mathbf{u}$ and $\mathbf{u}^{T}$ being the first column and first row, respectively. As a result, $\hat{\rho}$ is also expressed as

$$
\begin{aligned}
\hat{\rho} & =\frac{\left(\mathbf{x}_{31}-\mathbf{x}_{21}\right) \mathbf{W}_{S 1}\left(\mathbf{x}_{41}-\mathbf{x}_{11}\right)+\left(\mathbf{x}_{32}-\mathbf{x}_{22}\right) \mathbf{W}_{S 2}\left(\mathbf{x}_{42}-\mathbf{x}_{12}\right)}{\left(\mathbf{x}_{31}-\mathbf{x}_{21}\right) \mathbf{W}_{S 1}\left(\mathbf{x}_{31}-\mathbf{x}_{21}\right)+\left(\mathbf{x}_{32}-\mathbf{x}_{22}\right) \mathbf{W}_{S 2}\left(\mathbf{x}_{32}-\mathbf{x}_{22}\right)} \\
& =\frac{\left(\mathbf{x}_{31}-\mathbf{x}_{21}\right) \mathbf{W}_{S}\left(\mathbf{x}_{41}-\mathbf{x}_{11}\right)+r\left(\mathbf{x}_{32}-\mathbf{x}_{22}\right) \mathbf{W}_{S}\left(\mathbf{x}_{42}-\mathbf{x}_{12}\right)}{\left(\mathbf{x}_{31}-\mathbf{x}_{21}\right) \mathbf{W}_{S}\left(\mathbf{x}_{31}-\mathbf{x}_{21}\right)+r\left(\mathbf{x}_{32}-\mathbf{x}_{22}\right) \mathbf{W}_{S}\left(\mathbf{x}_{32}-\mathbf{x}_{22}\right)},
\end{aligned}
$$

where

$$
\mathbf{W}_{S}^{-1}=\operatorname{Toeplitz}\left(\left[\begin{array}{lllllll}
2\left(\rho^{2}+1\right) & -\rho^{2}-2 \rho & 2 \rho & -1 & 0 & \cdots & 0
\end{array}\right]\right) .
$$

As $\mathbf{W}_{S}$ is a function of the unknown $\rho$, the following iterative LLS procedure is employed to determine the frequency:

Step 1. Set $\mathbf{W}_{S}=\mathbf{I}_{N-3}$, which is the $(N-3)$ by $(N-3)$ identity matrix.

Step 2. Compute $\hat{\rho}$ using(8).

Step 3. Use $\rho=\hat{\rho}$ to construct $\mathbf{W}_{S}$ of (9).

Step 4. Repeat Steps 2 and 3 until a stopping criterion is reached.

Step 5. Compute the frequency estimate $\hat{\omega}$ as $\hat{\omega}=\cos ^{-1}((\hat{\rho}-1) / 2)$.

With the use of $\hat{\omega}$, the estimates of $\alpha_{i}, \phi_{i}$, and $C_{i}$, denoted by $\hat{\alpha}_{i}, \hat{\phi}_{i}$, and $\hat{C}_{i}$, respectively, are obtained by minimizing the following LLS cost function:

$$
(\boldsymbol{\Xi} \boldsymbol{K}-\mathbf{x})^{T}(\boldsymbol{\Xi} \boldsymbol{K}-\mathbf{x})
$$

where

$$
\boldsymbol{\Xi}=\operatorname{blkdiag}\left(\boldsymbol{\Xi}_{1}, \boldsymbol{\Xi}_{2}\right),
$$$$
\boldsymbol{\kappa}=\left[\boldsymbol{\kappa}_{1}^{T}, \boldsymbol{\kappa}_{2}^{T}\right]^{T},
$$

$\boldsymbol{\kappa}_{1}=\left[\alpha_{1} \cos \left(\phi_{1}\right) \alpha_{1} \sin \left(\phi_{1}\right) C_{1}\right]^{T}, \boldsymbol{\kappa}_{2}=\left[\alpha_{2} \cos \left(\phi_{2}\right) \alpha_{2} \sin \left(\phi_{2}\right) C_{2}\right]^{T}$,

$$
\begin{gathered}
\mathbf{x}=\left[\mathbf{x}_{1}^{T}, \mathbf{x}_{2}^{T}\right]^{T}, \\
\mathbf{x}_{1}=\left[x_{1}(1) x_{1}(2) \cdots x_{1}(N)\right]^{T}, \mathbf{x}_{2}=\left[x_{2}(1) x_{2}(2) \cdots x_{2}(N)\right]^{T}, \\
\mathbf{\Xi}_{1}=\mathbf{\Xi}_{2}=\left[\begin{array}{cccc}
\cos (\hat{\omega}) & \cos (2 \hat{\omega}) & \cdots & \cos (N \hat{\omega}) \\
-\sin (\hat{\omega}) & -\sin (2 \hat{\omega}) & \cdots & -\sin (N \hat{\omega}) \\
1 & 1 & \cdots & 1
\end{array}\right]^{T} \cdot(11)
\end{gathered}
$$

The LLS estimate of $\boldsymbol{\kappa}$ is

$$
\hat{\boldsymbol{\kappa}}=\left[\hat{\boldsymbol{\kappa}}_{1}^{T}, \hat{\boldsymbol{\kappa}}_{2}^{T}\right]^{T}=\left(\boldsymbol{\Xi}^{T} \boldsymbol{\Xi}\right)^{-1} \boldsymbol{\Xi}^{T} \mathbf{x},
$$

which gives $\hat{\alpha}_{i}=\sqrt{\left[\hat{\boldsymbol{\kappa}}_{i}\right]_{1}^{2}+\left[\hat{\boldsymbol{\kappa}}_{i}\right]_{2}^{2}}, \hat{\phi}_{i}=\tan ^{-1}\left(\left[\hat{\boldsymbol{\kappa}}_{i}\right]_{2} /\left[\hat{\boldsymbol{\kappa}}_{i}\right]_{1}\right)$, and $\hat{C}_{i}=\left[\hat{\boldsymbol{\kappa}}_{i}\right]_{3}, i=1,2$. Here, $\left[\hat{\boldsymbol{\kappa}}_{i}\right]_{l}, l=1,2,3$, denotes the $l$-th element of $\hat{\boldsymbol{\kappa}}_{i}$.

If the value of each DC offset is zero, the LP relation of (3) will be simplified and the number of unknowns is reduced to 5 . We can still apply the proposed methodology to perform accurate parameter estimation.

\section{Variance Analysis}

We now produce the variance of $\hat{\omega}$ based on small error conditions such that $\hat{\rho}$ is sufficiently close to $\rho$. For simplicity but without loss of generality, the noise variances $\sigma_{1}^{2}$ and $\sigma_{2}^{2}$ are set equal to $\sigma^{2}$. Let $\mathbf{z}_{i}=\overline{\mathbf{z}}_{i}+\Delta \mathbf{z}_{i}, i=1,2$, where $\overline{\mathbf{z}}_{i}$ and $\Delta \mathbf{z}_{i}$ are the noise-free version and perturbation of $\mathbf{z}_{i}$, respectively. Upon parameter convergence and applying Taylor series expansion, we obtain from (5)

$$
f(\hat{\rho})=\mathbf{z}_{2}^{T} \mathbf{W}\left(\mathbf{z}_{1}-\hat{\rho} \mathbf{z}_{2}\right)=0 \approx f(\rho)+f^{\prime}(\rho) \Delta \rho,
$$


where $\Delta \rho=\hat{\rho}-\rho$. Using $\overline{\mathbf{z}}_{1}=\overline{\mathbf{z}}_{2} \rho$ and neglecting secondorder perturbation terms, $f(\rho)$ can be rewritten as

$\left(\overline{\mathbf{z}}_{2}+\Delta \mathbf{z}_{2}\right)^{T} \mathbf{W}\left(\left(\overline{\mathbf{z}}_{1}+\Delta \mathbf{z}_{1}\right)-\rho\left(\overline{\mathbf{z}}_{2}+\Delta \mathbf{z}_{2}\right)\right) \approx \overline{\mathbf{z}}_{2}^{T} \mathbf{W}\left(\Delta \mathbf{z}_{1}-\Delta \mathbf{z}_{2} \rho\right)$.

As only first-order terms are retained, $f^{\prime}(\rho) \Delta \rho$ is approximated as

$$
f^{\prime}(\rho) \Delta \rho \approx-\overline{\mathbf{z}}_{2}^{T} \mathbf{W} \overline{\mathbf{z}}_{2} \Delta \rho .
$$

Based on (13) through (15), we have

$$
\Delta \rho \approx \frac{\overline{\mathbf{z}}_{2}^{T} \mathbf{W}\left(\Delta \mathbf{z}_{1}-\Delta \mathbf{z}_{2} \rho\right)}{\overline{\mathbf{z}}_{2}^{T} \mathbf{W} \overline{\mathbf{z}}_{2}} .
$$

The variance of $\hat{\rho}$, denoted by $\operatorname{var}(\hat{\rho})$, is obtained by squaring both sides of (16) and then taking the expected value:

$$
\begin{aligned}
\operatorname{var}(\hat{\rho}) & =E\left\{(\Delta \rho)^{2}\right\} \\
& \approx \frac{\overline{\mathbf{z}}_{2}^{T} \mathbf{W} E\left\{\left(\Delta \mathbf{z}_{1}-\Delta \mathbf{z}_{2} \rho\right)\left(\Delta \mathbf{z}_{1}-\Delta \mathbf{z}_{2} \rho\right)^{T}\right\} \mathbf{W} \overline{\mathbf{z}}_{2}}{\left(\overline{\mathbf{z}}_{2}^{T} \mathbf{W} \overline{\mathbf{z}}_{2}\right)^{2}} \\
& \approx \frac{\sigma^{2}}{\overline{\mathbf{z}}_{2}^{T} \mathbf{W} \overline{\mathbf{z}}_{2}} .
\end{aligned}
$$

Finally, the variance of $\hat{\omega}, \operatorname{var}(\hat{\omega})$, is obtained from (17) with the use of $\rho=(2 \cos (\omega)+1)$ [5]:

$$
\operatorname{var}(\hat{\omega}) \approx \frac{\operatorname{var}(\hat{\rho})}{4 \sin ^{2}(\omega)} \approx \frac{\sigma^{2}}{4\left(\overline{\mathbf{z}}_{2}^{T} \mathbf{W} \overline{\mathbf{z}}_{2}\right) \sin ^{2}(\omega)} .
$$

\section{Simulation Results}

Computer simulations are carried out to evaluate the proposed dual-channel sine-wave parameter estimator by comparing its MSE performance with the optimal benchmark of the CRLB as well as the NLS method [2]. We use the number of iterations as the stopping criterion in the iterative

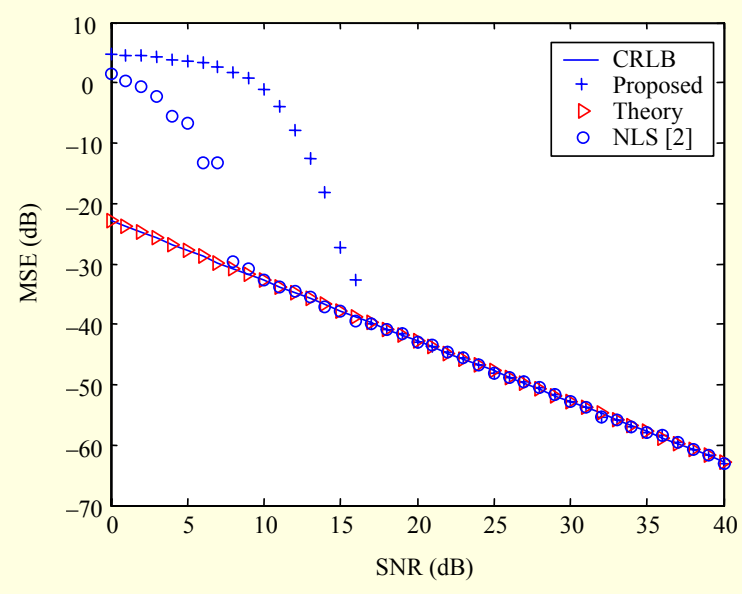

Fig. 1. MSE for frequency vs. SNR.
LLS algorithm, which is assigned as 5. For [2], $4 N$ uniformly-spaced grid points between $[0, \pi]$ are used to

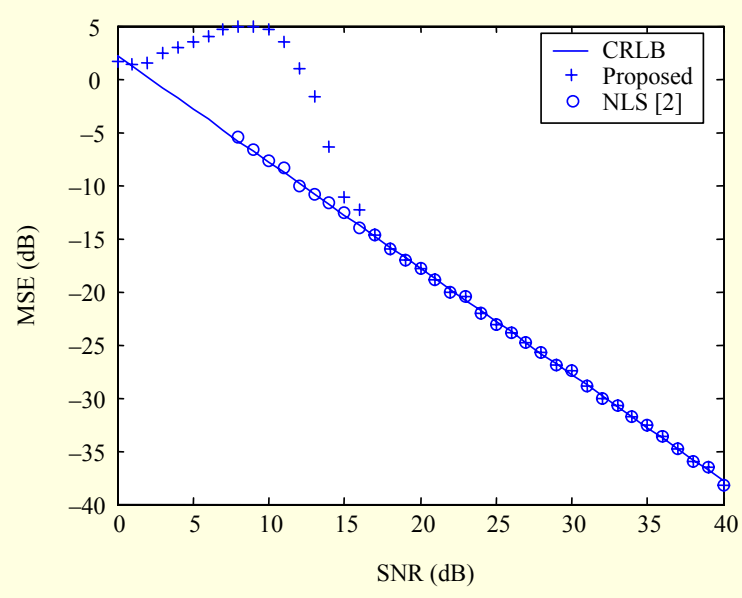

Fig. 2. Average MSE for amplitudes vs. SNR.

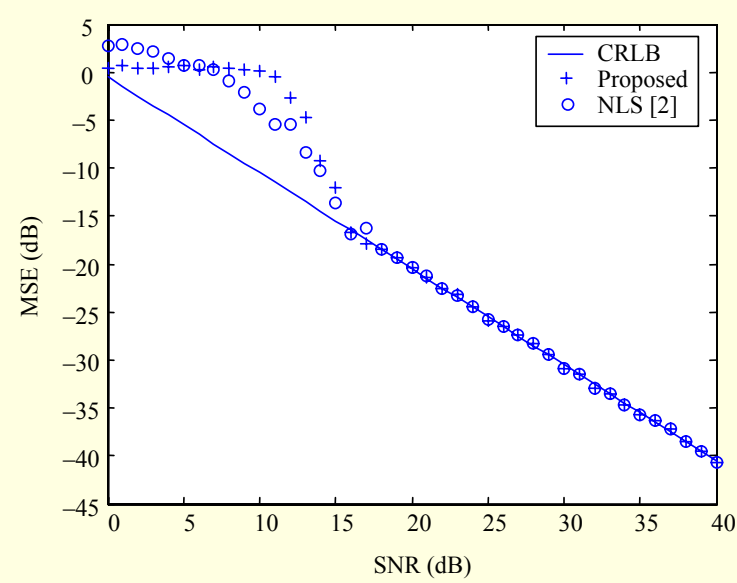

Fig. 3. Average MSE for phases vs. SNR.

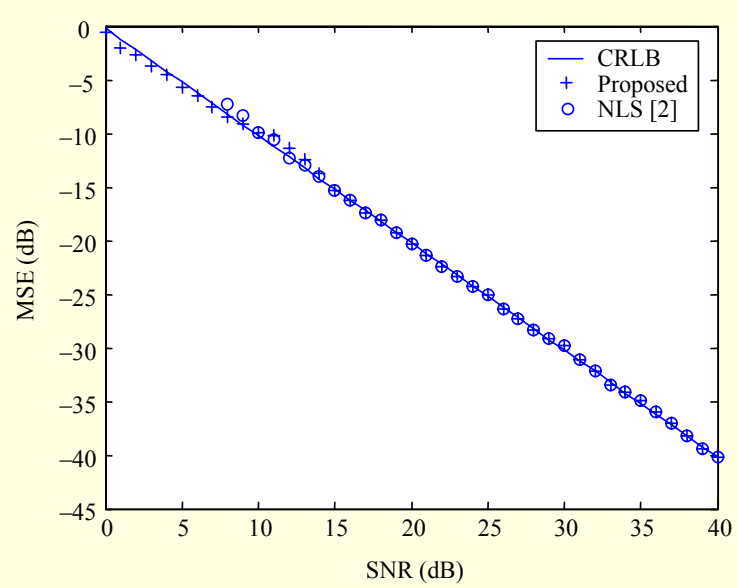

Fig. 4. Average MSE for offsets vs. SNR. 
obtain the coarse estimate while the golden section search is employed for final estimation. The parameter settings are $\omega=0.3, \quad \alpha_{1}=3, \quad \alpha_{2}=2, \quad \phi_{1}=1, \quad \phi_{2}=2, \quad C_{1}=2, C_{2}=2.5$, and $N=20$. We scale the white Gaussian noise sequences $v_{1}(n)$ and $v_{2}(n)$ to generate different SNR conditions, where $\mathrm{SNR}=\left(\alpha_{1}^{2} / 2+\alpha_{2}^{2} / 2+C_{1}^{2}+C_{2}^{2}\right) / \sigma^{2}$. For simplicity, the noise variances $\sigma_{1}^{2}$ and $\sigma_{2}^{2}$ are set as equal or $r=1$. All the results provided are averages of 500 independent runs.

Figures 1 through 4 show the MSEs for the frequency, amplitudes, phases, and offsets, respectively, at $\mathrm{SNR} \in[0,40] \mathrm{dB}$. It is seen that all the LLS parameter estimates attain the optimum accuracy at sufficiently high SNR, that is, when $\mathrm{SNR} \geq 16 \mathrm{~dB}$. In this SNR range, the empirical MSE value also agrees well with the theoretical calculation of (18), which is equal to the CRLB. On the other hand, we observe that the NLS method gives better threshold performance in frequency and amplitude estimation but it has much larger errors in the amplitudes and offsets at smaller SNRs. Note that increasing the number of grid points in [2] can enhance the threshold performance of the frequency estimate but at the expense of higher computational requirement.

\section{Conclusion}

An iterative LLS algorithm was devised to accurately estimate the parameters of dual-channel sinusoidal signals in white noise. The basic ideas are to utilize the LP property and LLS technique. The variance of the frequency estimate is derived and validated by simulations. It is also shown that the MSE performance of all parameter estimates attains CRLB in sufficiently high SNR conditions.

\section{References}

[1] T. Radil, P.M. Ramos, and A.C. Serra, "Impedance Measurement with Sine-Fitting Algorithms Implemented in a DSP Portable Device," IEEE Trans. Instrum. Meas., vol. 57, no. 1, Jan. 2008, pp. 197-204.

[2] P. Handel, "Parameter Estimation Employing a Dual-Channel Sine-Wave Model under a Gaussian Assumption," IEEE Trans. Instrum. Meas., vol. 57, no. 8, Aug. 2008, pp. 1661-1669.

[3] P.M. Ramos, F.M. Janeiro, and T. Radil, "Performance Comparison of Three Algorithms for Two-Channel Sinewave Parameter Estimation: Seven Parameter Sine Fit, Ellipse Fit, Spectral Sinc Fit," Proc. IMEKO World Congress, Lisbon, Portugal, vol. 1, Sept. 2009, pp. 480-484.

[4] F.K.W. Chan et al., "Accurate Parameter Estimation for Wave Equation," Prog. Electromagn. Research, vol. 102, 2010, pp. 3148.
[5] F.K.W. Chan et al., "Iterative Quadratic Maximum Likelihood Based Estimator for a Biased Sinusoid," Signal Process., vol. 90, no. 6, June 2010, pp. 2083-2086.

[6] F.K.W. Chan et al., "Efficient Approach for Sinusoidal Frequency Estimation of Gapped Data," IEEE Signal Process. Lett., vol. 17, no. 6, June 2010, pp. 611-614. 